Unfallchirurg 2013 $\cdot 116: 774-775$

DOI 10.1007/s00113-013-2381-6

Online publiziert: 6. September 2013

๑) Springer-Verlag Berlin Heidelberg 2013

\author{
S. Rammelt ${ }^{1} \cdot$ F. Gebhard ${ }^{2}$ \\ ${ }^{1}$ Klinik für Unfall- und Wiederherstellungschirurgie, Universitätsklinikum Carl Gustav Carus Dresden \\ ${ }^{2}$ Klinik für Unfallchirurgie, Hand-, Plastische und Wiederherstellungschirurgie, Zentrum für Chirurgie, \\ Universitätsklinikum Ulm
}

\title{
Oberes Sprunggelenk
}

Sowohl die ligamentären als auch die knöchernen Verletzungen des oberen Sprunggelenks (OSG) gehören zu den häufigsten ihrer Art am Bewegungsapparat und entsprechend groß ist das Interesse der unfallchirurgisch tätigen Kollegen an diesem Thema. Regelmäßig führen Sitzungen zu Sprunggelenkverletzungen und deren Folgen auch zu kontroversen Debatten auf wissenschaftlichen Kongressen. Die tägliche Erfahrung mit diesen Verletzungen lehrt uns, dass die Behandlungsergebnisse nicht immer den Erwartungen entsprechen.

\section{》) Der Strom neuer klinischer und biomechanischer Studien zum OSG reißt nicht ab}

Man möchte vielleicht meinen, dass $\mathrm{zu}$ diesem Themengebiet bereits alles ge sagt sei, aber der Strom neuer klinischer und biomechanischer Studien reißt nicht $\mathrm{ab}$ und in der aktuellen Literatur wird so ziemlich jedes lieb gewonnene Dogma hinterfragt. In vergangenen Weiterbildungsartikeln des Unfallchirurgen wurden verschiedentlich die chronischen Bandinstabilitäten, die Pathogenese und Akutversorgung von Sprunggelenkfrakturen sowie die Differentialindikation zur Arthrodese und Endoprothese bei fortgeschrittener Arthrose systematisch behandelt. Eine weitere Arbeit widmete sich speziell den Problemen und Kontroversen bei der Behandlung von Sprunggelenkfrakturen, von denen es beileibe nicht wenige gibt.

Im nun vorliegenden Themenheft werden spezielle Aspekte der Akutversorgung und der Behandlung von Spätfolgen der Bandverletzungen und Frakturen am
OSG herausgegriffen. So widmen sich $M$. Walther und sein Autorenteam in einem praxisorientiertem Beitrag dem ewig jungen Thema der Diagnostik und Behandlung sowohl der akuten als auch der chronischen Verletzungen des Außenbandapparats am OSG in übersichtlicher Weise.

Nachdem die Frakturen der Tibiahinterkante - historisch inkorrekt nach wie vor als „Volkmann-Dreieck“ bezeichnet jahrelang ein eher stiefmütterliches Dasein gefristet hatten und die Empfehlungen zur operativen Versorgung auf einer kleinen Fallserie aus den 1940er Jahren beruhten, hat sich in der letzten Dekade eine wahre Flut von Artikeln zu diesen über uns ergossen. D. Heim widmet sich diesem Thema in pragmatischer Weise und rechnet mit alten Dogmen ab, ohne gleich neue aufzustellen.

Residuelle Fehlstellungen nach Sprunggelenkfrakturen gehören leider ebenfalls zum klinischen Alltag und führen unbehandelt aufgrund der biomechanischen Gegebenheiten rasch zu einer posttraumatischen Arthrose. Jedoch können selbst bei schon erkennbaren Arthrosezeichen mit gelenkerhaltenden Korrektur osteotomien sehr gute Langzeitresultate erzielt werden, wie die dargestellten Erfahrungen aus Amsterdam und Dresden zeigen. In sorgfältig ausgewählten Fällen ist selbst nach Pilonfrakturen eine gelenkerhaltende Korrektur möglich.

Bei fortgeschrittener Arthrose stehen die Arthrodese und Endoprothese des OSG als z. T. konkurrierende, zum Großteil jedoch auch als ergänzende Verfahren zur Verfügung. Die Autoren um N. Espinosa gehen in ihrem Beitrag insbesondere auf die technischen Details und unbedingte Notwendigkeit der korrekten Ausrichtung bei beiden Verfahren ein.
Wir möchten uns bei allen Autoren für Ihr Engagement an diesem Heft bedanken und hoffen, dass die ausgewählten Themen Ihr Interesse finden.

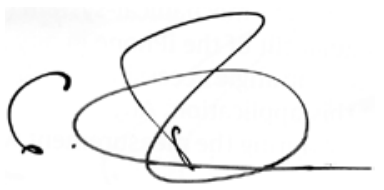

S. Rammelt

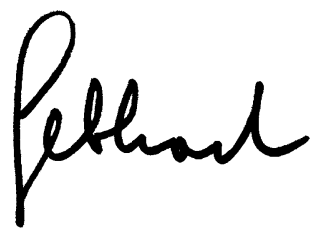

F. Gebhard

\section{Korrespondenzadressen}

\section{Prof. Dr. S. Rammelt}

Klinik für Unfall- und Wiederherstellungschirurgie, Universitätsklinikum Carl Gustav Carus Dresden Fetscherstraße 74, 01307 Dresden

stefan.rammelt@uniklinikum-dresden.de

\section{Prof. Dr. F. Gebhard}

Klinik für Unfallchirurgie, Hand-, Plastische und Wiederherstellungschirurgie,

Zentrum für Chirurgie, Universitätsklinikum Ulm Albert Einstein Allee 23, $89081 \mathrm{Ulm}$

florian.gebhard@uniklinik-ulm.de

\section{Einhaltung der ethischen Richtlinien}

Interessenkonflikt. F. Gebhard und S. Rammelt geben an, dass kein Interessenkonflikt besteht. 
Hier steht eine Anzeige.

黛 Springer 